loop length was related to the occurrence of livedo reticularis ( $\mathrm{p}$ $<0.05)$.

Conclusion Thus we suggest that nailfold capillaroscopy can be useful to better define SLE patients with APS, helping to identify some microvascular changes that seem related to clinical and laboratory features of APS.

\section{FRI0180 PARANEOPLASIC ANTIPHOSPHOLIPID SYNDROME- A POSSIBLE MAIN CAUSE OF THROMBOSIS IN CANCER PATIENTS}

C Tanaseanu, S Tanaseanu, A Urban, M Popescu, D Hanganu, A Cojan. Internal Medicine, Emergency Hospital "St. Pantelimon", Bucharest, Romania

10.1136/annrheumdis-2001.249

Background The antiphospholipid (APS) is a hypercoagulable state marked by the antibodies that recognise anionic phospholipid-protein cofactor complexes.

Objectives This study was undertaken to investigate a possible association of antiphospholipid antibodies (APLA) in cancer patients with thromboembolic events.

Methods The study group included 46 patients with different malignancies and associated thromboses. Tests were performed for detecting APLA: anticardiolipin enzyme ?linked immunoassay (ELISA) for both IgG and IgM isotypes, antiâ2-GPI IgG antibody ELISA, and coagulation test for lupus anticoagulant (LAC). We used ACL method with IL Test LAC Screen and IL Test LAC Confirm.

All patients underwent complete clinical and radiological examination, routine laboratory tests were done.

Results 18 patients had APS associated with evolutive cancers. LAC was present in 14 patients (B-cell lymphoma ( 2 cases), ovarian-(3), breast-(3), lung-(4), pancreatic-(1), bladder (1) cancer, and was related mainly with deep vein thromboses of the legs, pulmonary embolism and catastrophic antiphospholipid syndrome. Anticardiolipin antibodies (ACLA) were detected in 3 patients with bladder -, pancreatic - and breast cancer, and were associated with aortic nonocclusive thrombosis, stroke, myocardial infarction and multiinfarct dementia. In one patient (prostatic cancer) were present both ACLA and LAC, associated with stroke, myocardial infarction, multiinfarct dementia. The presence of LAC seems to be related more to venous thromboses and metastatic disease, while ACLA seems to be related to arterial thromboses.

Conclusion The presence of APS in cancer patients is associated with vascular thromboses, leading to a poor quality of life or to fatal events (including metastatic spread).

The presence of APLA in cancer patients may be a warning sign of a thrombotic event and might lead to a better selection of patients for prophylactic anticoagulant therapy.

\section{FRI0181 SKIN MANIFESTATIONS IN SECONDARY ANTIPHOSPHOLIPID SYNDROME - ANALYSIS OF FORTHY PATIENTS}

MM Cirkovic, DV Mitrovic, DZ Stefanovic, BC Glisic, MR Petronijevic, MM Popovic. Clinics of Rheumatology, Military Medical Academy, Belgrade, Yugoslavia

10.1136/annrheumdis-2001.250

Background Skin lesions are common kind of connective tissue disease manifestations.
Objectives The aim of study was evaluation of skin involvement in clinical presentation of 40 patients with systemic lupus erythematosus (SLE) and secondary antiphospholipid syndrome (APS).

Methods Examined group (34 women and 6 men) was represented by patients who fullfiled diagnostic ARA criteria for both, SLE and APS. Average duration of disease was $7.54 \pm$ 7.53 (1-33) years and average age of patients was $31.45 \pm$ 13.95 (8-65) years. Patients with SLE without APS represented control group.

Results We noticed cutaneous ulcerations in 15/40 (37.5\%) patients from examined group. They were predominantly located at lower legs and fingers of palms. Cutaneous ulcerations were significantly more frequently $(\mathrm{p}=0.025)$ appeared in patients with both, SLE and APS than in control group. Livedo reticularis was observed in $9 / 40(22.5 \%)$ patients from examined group, the most frequently at lower legs but one patient expressed livedo reticularis at skin of the torso. There was no significant difference in comparison to control group where prevalence of livedo reticularis was $10 \%(2 / 20)$. We observed palmar-plantar erythema in $12.5 \%(5 / 40)$ patients with APS as a part of SLE. In control group, palmar-plantar erythema was noticed in $10 \%$ patients $(2 / 20)$.

Conclusion Our study demonstrated that cutaneous ulcerations occurs more frequently in patients with both, SLE and APS than in patients with SLE alone, probably because of two present pathogenic mechanisms: vasculitis and thromophillia as a part od APS. On the other hand, livedo reticularis and palmar-plantar erythema were presented with the same frequency in both groups of patients.

\section{FRI0182 CATASTROPHIC ANTIPHOSPHOLIPID ANTIBODY SYNDROME - REPORT OF FIVE CASES}

P Barbosa, M França, I Almeida, F Farinha, C Vasconcelos. Department of Internal Medicine, Hospital Geral de Santo António, Porto, Portugal

10.1136/annrheumdis-2001.251

Background The catastrophic antiphospholipid antibody syndrome (CAPS) is an uncommon disorder, characterised by widespread micro and macrovascular changes due to intravascular thrombosis. We report five cases of CAPS occurred at our department over the past 7 years.

\section{Objectives}

Methods All patients were females between 13 and 49 years, all having SLE; two of them had no identifiable precipitant factor, two underwent major surgery, and the last one had a respiratory tract infection and a SLE flare as probable precipitant events; four women had deep vein thrombosis and the other one a peripheric arterial thrombosis; stroke occurred in 4 patients, one of them concomitantly in the right and left middle cerebral arteries terrritories; intraalveolar haemorrhage occurred in two cases and one patient developed ARDS.

Treatment options were different for each patient and all had steroids and anticoagulation. Two had cyclophosphamide synchronised with plasmapheresis.

Results Unfortunately 4 of the patients died, the only survivor was treated with steroids and anticoagulation therapy.

Conclusion CAPS is a rare event in our cohort of patients with antiphospholipid antibody syndrome (6,9\%), having a high mortality rate. Most of the times it was possible to identify a 